limiting case $n \mathrm{M} 0$. Actually, in (2a) we have retained the leading term while neglecting $O\left(n^{2}\right)$, which is consistent with the linear-perturbation theory.

$F(n)$ according to (2) and (2a) is plotted in Figure 1. It is clear that Göthert's and Sauer's conclusion cannot be correct in the range of practical interest $(1 / 10<n<1 / 3$ and $0.6 \leqq \beta<1$, say), since all of the various procedures listed above result in appreciable corrections to the velocity ratio. It seems more reasonable to conclude merely that the linear-perturbation theory cannot distinguish between the various'results. In this situation the formula of Method I might well be adopted by reason of its simplicity.

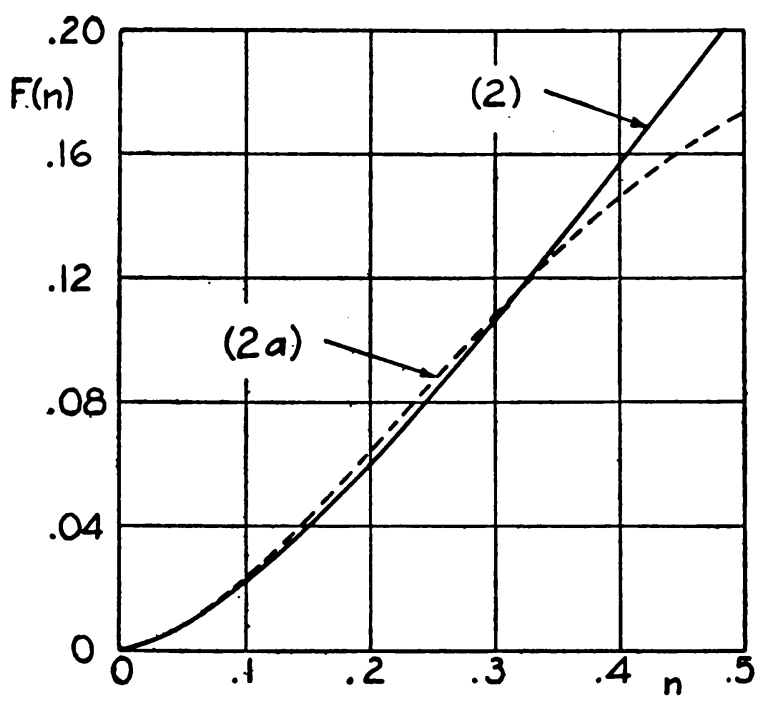

FIG. 1. The superstream velocity ratio for ellipsoids of revolution in incompressible flow.

\title{
ON THE NUMERICAL TREATMENT OF FORCED OSCILLATIONS*
}

\section{By ALVIN C. SUGAR** (Northrop Aircraft)}

1. Introduction. The differential equation, with typical initial conditions, of an harmonic oscillator subject to the action of a general disturbing force $m a(t)$ is given by

$$
\ddot{x}+\omega^{2} x=a(t), \quad x(0)=0=\dot{x}(0) .
$$

This equation occurs in problems involving from one to infinitely many degrees of freedom. Its solution can be expressed as follows:

$$
x=\frac{D}{\omega}, \quad \text { where } \quad D=\int_{0}^{t} a(\tau) \sin \omega(t-\tau) d \tau
$$

is the so-called Duhamel integral. If in (1) we replace only $x$ by $D / \omega$ we obtain an expression for the acceleration of the body.

$$
\ddot{x}=a(t)-\omega D .
$$

In this note a simple expression which is an approximation of $D$ is found. This expression provides a convenient process for evaluating $x$ and related quantities. Using the resulting simplified form of the acceleration a quick and easy vector method of obtaining the maximum acceleration is explained. Rapid methods of finding the

* Received Oct. 1, 1945.

** Now at Brown University. 
maximum displacement are also considered. By maximum acceleration is meant maximum magnitude or absolute value of acceleration and similarly for displacement.

2. Evaluation of the Duhamel integral. The curve $y=a(t)$ is approximated by a broken line $Y=A(t)$, see Fig. 1 . As indicated we are taking $A\left(t_{0}\right)=0$. Since the approximation of a curve by a broken line can be improved by increasing the number of segments, it is evident that this method can produce a solution which is as accurate

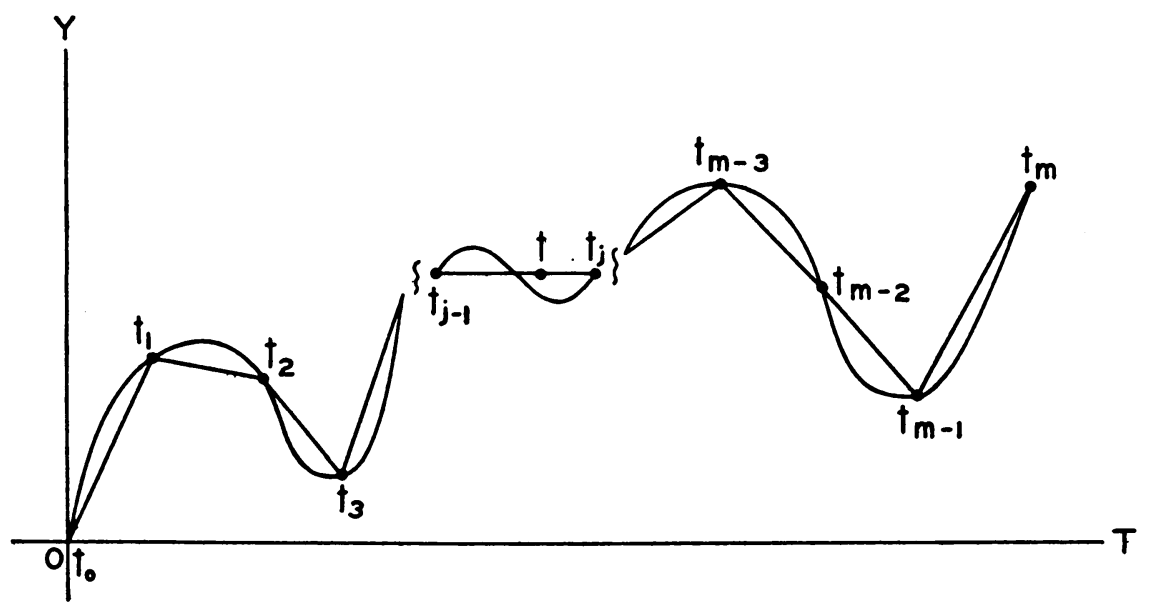

FIG. 1

as desired. Frequently in engineering problems the forcing function is known only approximately and the additional error introduced by a broken line consisting of relatively few segments is negligible.

Let $A(t)=A_{i}(t), t_{i-1} \leqq t \leqq t_{i}$, where $y_{i}=A_{i}(t)$ is the equation of a straight line of slope $\mu_{i}$. Since the lines $y_{i}=A_{i}(t)$ and $y_{i+1}=A_{i+1}(t)$ intersect at the point $\left(t_{i}, A_{i}\left(t_{i}\right)\right)$, it follows that

$$
A_{i+1}\left(t_{i}\right)=A_{i}\left(t_{i}\right) .
$$

With the notations of Fig. 1 the Duhamel integral can be approximated in the following manner:

$$
\begin{aligned}
D & =\int_{0}^{t} a(\tau) \sin \omega(t-\tau) d \tau \\
& \approx \sum_{i=1}^{j-1} \int_{t_{i-1}}^{t_{i}} A_{i}(\tau) \sin \omega(t-\tau) d \tau+\int_{t_{j-1}}^{t} A_{j}(\tau) \sin \omega(t-\tau) d \tau .
\end{aligned}
$$

Integration by parts enables us to write this in the form

$$
\omega D=A(t)-\frac{1}{\omega}\left[\sum_{i=1}^{j} \mu_{i} \sin \omega\left(t-t_{i-1}\right)-\sum_{i=0}^{j-1} \mu_{i} \sin \omega\left(t-t_{i}\right)\right]
$$

where we have defined $\mu_{0}=0$ in order to obtain the following compact result:

$$
\omega D=A-\frac{1}{\omega}\left[\sum_{i=0}^{j-1}\left(\mu_{i+1}-\mu_{i}\right) \sin \omega\left(t-t_{i}\right)\right] .
$$


From (3) and (5) it is evident that

$$
\ddot{x} \approx A-\omega D=\frac{1}{\omega} \sum_{i=0}^{i-1}\left(\mu_{i+1}-\mu_{i}\right) \sin \omega\left(t-t_{i}\right) .
$$

3. Maximum acceleration and displacement. It is, at times, of importance to know the maximum displacement or acceleration. In this paragraph we show a vector method of obtaining the maximum acceleration. Since the expression for $\ddot{x}$ for the $j$ th time interval is, apart from a constant factor, a sum of sinusoids of the same frequency, $\omega$, it is equal to a sinusoid of frequency $\omega$. The amplitude of this sinusoid can be obtained by vector methods. It is evident that this amplitude is equal to the maximum of the absolute value of the resultant sinusoid over a time interval equal to or in excess of one half period. From this it is apparent that the following vector procedure can be used in determining max $|\ddot{x}|$ over all of the time intervals.

If

$$
\frac{\pi}{\omega} \leqq \min \left(t_{i+1}-t_{i}\right), \quad i=0,1, \cdots, m,
$$

then $\max |\ddot{x}|=\max O P_{i}$ (Fig. 2), where the magnitude of the $i$ th vector is $\left|\left(\mu_{i}-\mu_{i-1}\right) / \omega\right|$ and its argument with respect to $O P_{1}$ is equal to the phase angle $-\omega t_{i-1}$.

Without condition (7) max $O P_{i}$ is an upper bound of $|\ddot{x}|$ and probably a pretty good approximation of $\max |\ddot{x}|$.

Fairly rapid methods of computing the maximum displacement ${ }^{1}$ can be devised e.g. when the frequency is large, then, for the $i$ th interval, $\max |x| \approx\left(1 / \omega^{2}\right)\left[\max \left|A_{i}(t)\right|\right.$ $+\max |\ddot{x}|]$. For any frequency the problem of finding max $|x|$ may be reduced to that of finding the maximum value of the curve obtained by the superposition of a sinusoid and a straight line. This can be handled by obvious methods involving use of elementary differential calculus.

A still better method of approximating the maximum displacement is available if $s$ is known or can be quickly evaluated, where $s$

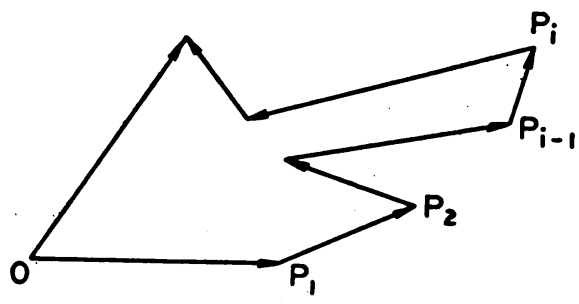

FIG. 2 is defined by the differential equation,

$$
\ddot{s}=a(t), \quad \dot{s}(0)=0=s(0) .
$$

This method permits direct use of the above vector procedure. This is easily shown by evaluating the Duhamel Integral by repeated integration by parts. ${ }^{2}$ Thus,

$$
\begin{aligned}
x & =\frac{1}{\omega} \int_{0}^{t} \ddot{s} \sin \omega(t-\tau) d \tau=s-\omega \int_{0}^{t} s \sin \omega(t-\tau) d \tau \\
& \approx \frac{1}{\omega} \sum_{k=0}^{j-1}\left(\nu_{k+1}-\nu_{k}\right) \sin \omega\left(t-t_{k}\right),
\end{aligned}
$$

${ }^{1}$ A mechanical analyzer was invented by M. A. Biot to obtain this maximum [Bulletin Seismological Soc. Amer. 31, 151-171 (1941)].

2 As was done by G. W. Housner in obtaining his formulas (2) and (3), Bulletin Seismological Soc. Amer. 31, 143-149 (1941). 
where $\nu_{k}$ and $t_{k}$ are defined by a broken line approximation of $s$. If the initial conditions of (8) defining $s$ are changed to $\dot{s}(0)=s_{0}$ and $s(0)=s_{0}$, then (9) takes the following form:

$$
x=-\frac{\dot{s}_{0}}{\omega} \sin \omega t-s_{0} \cos \omega t+s-\omega \int_{0}^{t} s \sin \omega(t-\tau) d \tau,
$$

which again can be expressed as a sum of sinusoids of the same frequency.

On the basis of limited experience, the following suggestions for computation seem good. If the curve is sufficiently smooth, then the term containing $\mu_{1}$ will make a sizeable contribution; consequently the first time interval should be as small as convenient. It seems best to take $A(0)=a(0)$. The vector polygon will obviously be simplest if $t_{i}$ is selected so that as many values as possible of $\omega t_{i}$ are multiples of $\pi$.

If we had assumed $A(0) \neq 0$, then it would follow that

$$
\omega D=A-A(0) \cos \omega t-\frac{1}{\omega} \sum_{i=0}^{j-1}\left(\mu_{i+1}-\mu_{i}\right) \sin \omega\left(t-t_{i}\right) .
$$

If we let $\dot{s}_{k}=\dot{s}\left(t_{k}\right)$ then it is clear that $\nu_{k+1} \approx \xi_{k+1}$. Substituting in (9) we have

$$
x \approx \frac{1}{\omega} \sum_{k=0}^{j-1}\left(\xi_{k+1}-s_{k}\right) \sin \omega\left(t-t_{k}\right) .
$$

In calculating the maximum displacement, (11) would be more convenient than (9) since $s$ could be obtained by a single integration of $a(t)$.

\title{
A REMARK ON THE RECTIFICATION OF THE JOUKOWSKI PROFILE*
}

\author{
BY CHARLES SALTZER (Brown University)
}

The Joukowski profile is usually defined as the image under the Joukowski transformation,

$$
\zeta=z+c^{2} / z
$$

of a circle passing through the point $(-c, 0)$ whose center lies in the first quadrant, and whose radius is $c(1+\epsilon)$ where $c$, and $\epsilon>0$. Although this representation gives the complex potential of the incompressible flow about a Joukowski profile very readily, the representation of this profile as the inverse of a parabola ${ }^{1}$ has the advantage, as will be shown below, of introducing a parameter with direct geometrical meaning which permits the immediate rectification of the Joukowski profile in closed form.

In the $z_{1}$-plane consider the parabola

$$
y_{1}=\frac{1}{2} x_{1}^{2}
$$

It is interesting to note that the sum in (11) is the so-called left Cauchy-Stieltjes sum corresponding to $D$.

* Received Aug. 17, 1945.

'In this way the profile later called "Joukowski profile" was introduced by Chaplygin. See Chapylgin's Collected Papers, Leningrad 1933, vol. 2, pp. 144-178, in particular $\$ 6$. 